\title{
Die transnationale Klasse - Mythos oder Realität?
}

\author{
Von Michael Hartmann
}

Zusammenfassung: In der Diskussion über die Inter- und die Transnationalisierung von Klassen und Eliten gehen die Meinungen weit auseinander. Der Artikel beschäftigt sich mit diesem Thema anhand einer empirischen Untersuchung über die Nationalität und die Karrieren der Topmanager der fünf größten europäischen (Deutschland, Frankreich, Großbritannien, Italien und Spanien) und der drei größten außereuropäischen Wirtschaftsmächte (China, Japan und USA). Das Ergebnis ist eindeutig. Nur jeder zwanzigste Spitzenmanager kommt aus dem Ausland und nur jeder vierte einheimische Topmanager hat jemals im Ausland studiert oder gearbeitet. Länger als zwei Jahre im Ausland hat sogar nur jeder siebte einheimische Topmanager gelebt. Der wesentliche Grund dafür ist die ungebrochene Vorherrschaft der traditionellen nationalen Karrieremuster. Die Inter- und Transnationalisierung der Wirtschaftseliten geht bedeutend langsamer voran als viele Soziologen und Wirtschaftwissenschaftler vermuten. Es gibt allerdings große Unterschiede zwischen den einzelnen Ländern. In Deutschland und Großbritannien ist der Prozess am weitesten vorangeschritten. In Frankreich ist in der jüngeren Generation dagegen sogar ein Rückgang zu verzeichnen.

\section{Einleitung}

Zwei der prominentesten deutschen Nachkriegssoziologen, Ralf Dahrendorf und Ulrich Beck, sind in jüngster Zeit mit sehr unterschiedlichen Äußerungen zum Thema Transnationalisierung in die wissenschaftliche Öffentlichkeit getreten. Dahrendorf nimmt in einem Interview mit dem „Leviathan“ seine vor knapp einem Jahrzehnt getroffene Feststellung von der Entstehung und der beginnenden Herrschaft einer ,globalen Klasse“ (Dahrendorf 2000: 1058ff) zumindest für die Gegenwart zurück. Auf die Frage, ob es diese globale Elite noch gebe oder sie sich zerstreue, antwortet er ganz unmissverständlich: „Die zerstreut sich im Augenblick sehr stark“ (Dahrendorf 2009: 178). Ganz anders Beck in einem Beitrag für die „Soziale Welt“ Ende letzten Jahres. Er spricht in seinem vehementen Plädoyer für eine Überwindung des methodischen Nationalismus davon, dass ,neuartige radikalisierte Ungleichheitshierarchien jenseits von Nation und Klasse“ entstünden, das „reiche obere Drittel der Welthierarchie“ und vor allem die reichen Eliten eine „Polygamie des Ortes“ praktizierten. Diejenigen, die über das entsprechende ökonomische Kapital oder kulturelle Kapital verfügen, „um den optimalen Kontext für deren Verwertung selbst zu wählen“, befänden sich einfach in einer besseren Position als diejenigen, die z.B. aus wohlfahrtsstaatlicher Bindung an bestimmte Arbeitsplätze an den nationalstaatliche Rahmen gebunden seien (Beck 2008: 316 f). Diese beiden Äußerungen zeigen, wie weit die Ansichten über die Inter- oder Transnationalisierung der Eliten mittlerweile selbst bei jenen auseinander gehen, die im Grunde eine ähnliche Position teilen.

Dieser Differenz auf der Ebene der soziologischen Analyse und Prognose entspricht auch eine Differenz in der alltäglichen Wahrnehmung. Man kann in den letzten Monaten eine erstaunliche und auf den ersten Blick sehr widersprüchliche Entwicklung beobachten. Auf der einen Seite gewinnt der Nationalstaat unübersehbar ganz erheblich an Bedeutung zurück. ,The state is back" titeln angelsächsische Wirtschaftsblätter, wenn sie die über die tiefgreifende Finanz- und Wirtschaftskrise schreiben. Das ManagerMagazin formuliert es noch schärfer „Der Staat schlägt zurück.“ Der Glaube an die Funktionsfähigkeit und die Regelungsmechanismen des Marktes hat durch die Krise stark gelitten, wird von vielen sogar grundsätzlich infrage gestellt. Überall muss der Staat einspringen, um den Kollaps des Finanzsystems zu verhindern. Die Zentralbanken haben inzwischen weit mehr als eine Billion Euro bzw. Dollar in die Märkte gepumpt, um den Finanzkreislauf wieder in Schwung zu bringen. Gleichzeitig sind die einzelnen Nationalstaaten mit ebenfalls weit über einer Billion Euro bzw. Dollar, sei 
es in Form von Bürgschaften, sei es in Form von Beihilfen oder direkten Beteiligungen, ihren maroden Banken beigesprungen. Allein die Hypo Real Estate muss von der Bundesregierung mittlerweile mit Garantien von mehr als 100 Mrd. Euro vor dem Ruin bewahrt werden. Islands Regierung hat die drei großen Banken des Landes sogar komplett verstaatlichen müssen, um sie vor dem Untergang zu retten. Sie hinterlassen einen Schuldenberg vom Zehnfachen der jährlichen Wirtschaftsleistung des Landes. So weit ein eindeutiges Bild. Die Ära der kaum regulierten Märkte, der ungebremsten Globalisierung und des massiven Bedeutungsverlustes der Nationalstaaten scheint vorbei.

Dieser Eindruck kontrastiert allerdings merkwürdig mit der Tatsache, dass gleichzeitig die internationale Mobilität der Investmentbanker nicht gelitten zu haben scheint. Sie, die Speerspitze, wenn es um die Internationalisierung der Finanzmärkte geht, agieren weitgehend wieder so wie vor dem Crash. Das gilt nicht nur für ihre tägliche Arbeit, sondern, und das ist hier interessanter, auch in Hinblick auf ihre Neigung, das Unternehmen und / oder das Land ihrer beruflichen Tätigkeit zu wechseln. Es ist ein regelrechter Abwerbungswettlauf unter den großen Banken zu beobachten. Diejenigen unter ihnen, denen aufgrund massiver staatlicher Hilfen Einschränkungen bei den Boni-Zahlungen an ihre Topkräfte auferlegt worden sind, müssen erleben, wie die besten Investmentbanker von der Konkurrenz mit großzügigeren finanziellen Angeboten abgeworben werden. Das gilt für die Citigroup und die Bank of America genauso wie für Morgan Stanley oder die UBS. Sie versuchen dem etwas entgegen zu setzen, indem sie die Grundgehälter stark anheben. Gerade jene Banken, die auf staatliche Hilfe ganz besonders angewiesen sind, unterlaufen damit alle Forderungen nach einer ,neuen Bescheidenheit".

Ähnliches gilt auch für die Spitzenmanager. So hat der neue CEO der nach einem Jahresverlust von 24 Mrd. Pfund Sterling zu 70 Prozent verstaatlichten Royal Bank of Scotland, Stephen Hesters, einen Vertrag unterschrieben, der bei Erfüllung bestimmter Zielvorgaben ein Jahreseinkommen von bis zu 11,1 Mio. Euro vorsieht. Selbst bei den deutschen Landesbanken, die mit einem insgesamt höheren zweistelligen Milliardenbetrag vor dem Zusammenbruch bewahrt worden sind, kann man dasselbe Spiel beobachten. Der neue Vorstandschef der mit über 12 Mrd. Euro vom Land gestützten Landesbank Baden-Württemberg, Hans-Jörg Vetter, soll deutlich mehr verdienen als sein in Folge der Krise zurück getretener Vorgänger Siegfried Jaschinski. Der verdiente 700.000 Euro plus Zulagen. Vetter aber erhielt bei der Berliner Bank 2008 bereits rund 1,1 Mio. Euro Festgehalt plus Boni und Sonderzahlungen von 700.000 Euro. Das will er wenigstens bekommen. Die Landesregierung riskiert dafür sogar einen Verstoß gegen den eindeutigen Beschluss des Landtags, die Gehälter bei der LBBW auf maximal 500.000 Euro zu begrenzen. Auch bei der von den Bundesländern Hamburg und SchleswigHolstein mit einer Kapitalspitze von über drei Mrd. Euro direkt gestützten HSH-Nordbank erhält der Vorstandschef Dirk Jens Nonnenmacher trotz eines Beschlusses der Landesparlamente, die Gehälter auf 500.000 Euro zu begrenzen, eine Sondervergütung in Höhe von fast drei Mio. Euro. Das Argument für all diese Beschlüsse kommt einem aus früheren Jahren seltsam vertraut vor. Die heftige Konkurrenz auf dem globalen Markt für Spitzenmanager zwinge zu solchen Zahlungen, so der einstimmige Tenor bei Bankmanagern und Politikern. Typisch dafür ist eine Äußerung vom Vorstandsvorsitzenden der Deutschen Bank, Josef Ackermann, bei einer Podiumsdiskussion an der European School of Management and Technology (ESMT) Anfang Juli. Ackermann sagte dort zur Frage der Boni: „Wenn wir keine Boni zahlen, werden die guten Leute abgeworben. Ich weiß nicht, wie viele Leute ein internationales Unternehmen leiten können. Lassen Sie es zehn bis zwanzig Leute weltweit sein - dann zahlst du lieber den Preis“" (Financial Times Deutschland, 2.7.2009). 


\section{Die sozialwissenschaftliche Diskussion über die transnationale Elite}

So pointiert die Äußerungen von Beck und Dahrendorf zur „globalen Klasse“ oder zu den transnationalen „reichen Eliten“ ausfallen, so ungenau bleiben ihre Definitionen. Das gilt vor allem für Beck. So bleibt völlig unklar, wie er das ,reiche obere Drittel“ bestimmt. In seiner Argumentation bezieht es sich sehr stark auf die Begrifflichkeit von Weiß. Er übernimmt ihre Aussage von den ,upper und some upper middle layers of world society“ als Kernpunkt seiner Definition (Beck 2008: 316). Wenn man Weiß jedoch ernst nimmt, kommt man nicht einmal annähernd auf ein Drittel der Weltbevölkerung. ${ }^{1}$ Noch unschärfer wird das Ganze, wenn Beck in ein und demselben Satz erst vom ,reichen Drittel“ und dann übergangslos von den ,reichen Eliten" spricht.

Dahrendorfs Erklärung ist da schon präziser. Er schätzt, dass nicht mehr als ein Prozent der Bevölkerung zu der neuen „globalen Klasse“ gehört (Dahrendorf 2000: 1059). Das entspricht rein quantitativ dem, was die angelsächsische Sozialforschung traditionell als Upper Class bezeichnet. Dahrendorfs Darstellung lässt allerdings offen, inwieweit seine „Global Class“ mit der Upper Class identisch ist und wo sie sich von ihr unterscheidet. Die diesbezüglichen Ausführungen fallen ausgesprochen unscharf aus. Auf der einen Seite bezieht er sich auf Kanther und ihren Begriff der „Weltklasse“. Bei Kanther ist damit eine globale Elite bestehend aus den Topmanagern der führenden multinationalen Konzerne gemeint (Kanther 1995). Auf der anderen Seite spricht er dann aber von jungen Computerfreaks, die innerhalb weniger Wochen Millionen mit Börsengängen verdient hätten, Medienmachern und ,sogar einigen Professoren“, Stars in allen Lebensbereichen, vom Sport und der Musik bis hin zu Lotteriegewinnern. Geld scheint hier das wesentliche Kriterium für die Zugehörigkeit zu sein. Im nächsten Abschnitt setzt Dahrendorf die ,globale Klasse“ dann auch unmittelbar mit den ,neuen Millionären" gleich. Was mit den alten Millionären oder den Topmanagern ist, sagt es nicht. Alles bleibt seltsam verschwommen.

Der eindeutige Bezug auf Kanther bzw. Weiß lässt aber vermuten, dass sowohl Dahrendorf als auch Beck die Wirtschaftselite mit zum Kern dessen rechnen, was sie als globale Klasse oder transnationale Elite bezeichnen. Wirft man einen Blick auf aktuelle Studien über diese kleine Gruppe, dann wird schnell klar, dass die Differenz zwischen Beck und Dahrendorf nicht allein auf ihre unscharfen Definitionen zurückzuführen ist. Auch empirisch genaue Untersuchungen über diese Personen gelangen zu sehr unterschiedlichen Schlussfolgerungen und Prognosen, wenn es um die Frage nach der Transnationalität der Wirtschaftselite geht. Sehr deutlich zeigen das die jüngsten Veröffentlichungen von Yoo und Lee über Elitenetzwerke in Frankreich und Südkorea (Yoo / Lee 2009) sowie von Carroll über die transnationalen Netzwerke der ,interlocking directors“ in den 500 größten Unternehmen der Welt (Carroll 2009).

Carroll, der die Verbindungen unter den Board-Mitgliedern² dieser 500 Konzerne in den Jahren 1996 und 2006 untersucht hat, kommt zu zwei entscheidenden Aussagen. Erstens stellt er fest, dass man mit Recht von einer „global corporate elite“ sprechen könne (Caroll 2009: 295ff). Zwischen 1996 und 2006 habe sich das Netzwerk der Spitzenmanager intensiviert und

1 Weiß definiert ihren Begriff von den ,transnational upper classes“ zwar auch nicht genauer. Da sie bei ihren Ausführungen aber direkt an Sklair mit seiner ,,transnational capitalist class“ (Sklair 2001) anknüpft und danach als Erläuterung nur noch die Spitzenmanager der großen Unternehmen und die Geschäftsleute von der Semi-Peripherie benennt, dürfte sie alles in allem eher einen ziemlich kleinen Teil der Bevölkerung, in dessen Kern sich die auch traditionell unter den Begriff der Upper Class fallenden Kreise befinden, meinen als fast ein Drittel der Weltbevölkerung (Weiß 2005: Seite $713 \mathrm{ff}$ ).

2 In den angelsächsischen Ländern sind im Board die Funktionen des deutschen Vorstands und Aufsichtsrats verknüpft. Carroll hat dementsprechend sämtliche Personen in die Untersuchung einbezogen, die unabhängig von der konkreten Organisationsform die Funktionen von Board Members wahrnehmen. 
nicht gelockert. Das gelte besonders für die „Transnationalists“. Verglichen mit den ,national networkers" sei ihr Anteil an den Verbindungen massiv um ein Drittel gestiegen und sie bildeten auch ein eigenes, von den weiterhin existierenden nationalen Netzwerken unabhängiges und zugleich enger werdendes Netzwerk. Ihr Einfluss sei damit erheblich gewachsen. Zweitens, und das als Einschränkung, sei das Netzwerk der „Transnationalists“ jedoch kein wirklich globales, sondern im Kern ein regionales nordatlantisches, das sich auf Nordamerika und Europa erstrecke. Dort allerdings sei es hoch integriert (ebd.: 305ff). Im Unterschied zu Beck, Dahrendorf und einer Vielzahl anderer Autoren, die mit Begriffen wie globale oder transnationale Elite relativ schnell bei der Hand sind, weist Carroll als sorgfältiger Empiriker in seinem Schlusskapitel auch auf die Grenzen seiner Analyse hin. Er betont dort ausdrücklich, dass Aussagen von einer national nicht mehr verwurzelten, sondern nur in einem transnationalen Raum agierenden Wirtschaftselite die Beharrlichkeit nationaler und regionaler Bindungen unterschätzten. Die nationalen Netzwerke bildeten immer noch das Rückgrat der ,global corporate elite". Der Blick auf letztere sei allerdings entscheidend, wolle man die Frage nach der Formierung einer „transnational capitalist class“ beantworten (Carroll 2009: $308 \mathrm{f}$ ).

Yoo und Lee betonen den genau entgegen gesetzten Punkt. Sie zeigen, welch große Bedeutung in Frankreich und Südkorea nach wie vor den nationalen Elitenetzwerken zukommt. In beiden Ländern, so ihre Analyse, dominierten diese weiterhin ganz eindeutig. Sie seien im Kern geprägt durch die gemeinsame Ausbildung ihrer Mitglieder an einigen, wenigen Elitehochschulen des jeweiligen Landes und durch enge Verbindungen zwischen den wirtschaftlichen und den staatlichen Eliten, was die Karrierewege und die Kooperation der Eliten angehe (Yoo / Lee 2009: 536ff). Von einer Transnationalisierung von Elitenetzwerken ist bei Yoo und Lee dagegen keine Rede.

Im Folgenden werden empirische Ergebnisse präsentiert, die zur Klärung der Differenzen und Widersprüche beitragen und eine besser fundierte Aussage zur Frage der transnationalen Elite ermöglichen sollen. Zu diesem Zweck wird der Blick auf die Bildungs- und Berufskarrieren all jener Personen gerichtet, die an der Spitze eines der führenden Unternehmen in den wichtigsten Ländern Europas, Ostasiens und Nordamerikas stehen. Es sind dies die die Spitzenmanager (CEO, Chairmen, Presidents, PDG oder Vorstandsvorsitzende) der fünf größten europäischen (Deutschland, Frankreich, Großbritannien, Italien und Spanien) und der drei größten außereuropäischen Wirtschaftsmächte (China, Japan und die USA). Diese acht Länder stellen drei Viertel der 500 größten Unternehmen der Welt. Für China, Deutschland, Frankreich, Großbritannien, Japan und die USA sind die 100 größten Konzerne, für Italien und Spanien aufgrund ihrer deutlich geringeren Bedeutung in der Weltwirtschaft nur die 50 bzw. die 30 größten in die Untersuchung einbezogen worden. Die Unternehmen sind anhand der einschlägigen Ranglisten (FAZ, Financial Times, Forbes, Fortune, l'Expansion und Xinhua) für die Jahre 2005 (Deutschland, Frankreich, Großbritannien und die USA), 2006 (Italien, Japan und Spanien) sowie 2007 (China) ermittelt worden. Für China, Italien und Spanien sind die Listen unverändert übernommen worden, für Deutschland, Frankreich, Großbritannien, Japan und die USA sind sie, um vergleichen zu können, nach dem gleichen Muster wie in einer älteren Untersuchung (Hartmann 1999) gegliedert worden. Maßstab ist nicht der Börsenwert, sondern der Umsatz bzw. die Bilanz- oder Versicherungssumme und die Aufteilung erfolgt nach folgendem Prinzip: 50 Prozent Industrie, 30 Prozent Handel und Dienstleistungen sowie 20 Prozent Banken und Versicherungen. Für Großbritannien ist die Finanzbranche aufgrund 
der enormen Bedeutung des Finanzplatzes London mit 25 Prozent etwas stärker gewichtet worden. ${ }^{3}$

\section{Ausländer - eine kleine Minderheit in der Wirtschaftselite}

Der erste Befund ist ganz eindeutig. Die führenden Unternehmen dieser Länder werden fast immer von Einheimischen geführt (siehe Tabelle 1). Nimmt man alle acht Länder zusammen, so liegt der Anteil der Ausländer, die einen der jeweils 100 größten Konzerne dieser Länder leiten, insgesamt bei nicht mehr als gut fünf Prozent. Lässt man jene Topmanager außer Betracht, die demselben Sprach- und Kulturkreis entstammen, wie die Schweizer und Österreicher in Deutschland, die Australier, Iren, Kanadier und Südafrikaner in Großbritannien und den USA sowie zusätzlich die US-Amerikaner in Großbritannien, dann reduziert sich der Prozentsatz sogar auf nur noch gut zwei Prozent. Von einer Internationalität des Topmanagements kann selbst in den großen, international führenden multinationalen Konzernen keine Rede sein.

Tabelle 1: Internationalität der Topmanager (Vorstandsvorsitzende, CEO, Chairmen, PDG, Presidents) der 100 größten Unternehmen Deutschlands, Frankreichs, Großbritanniens, Chinas, Japans und der USA sowie der 50 größten Unternehmen Italiens und der 30 größten Unternehmen Spaniens (in Prozent)

\begin{tabular}{|c|c|c|c|c|c|c|c|c|}
\hline & $\begin{array}{c}D \\
n=100\end{array}$ & $\begin{array}{c}F \\
n=100\end{array}$ & $\underset{n=100}{\text { GB }}$ & $\begin{array}{c}\text { IT } \\
\mathbf{n}=\mathbf{5 0}\end{array}$ & $\begin{array}{c}\text { ESP } \\
\mathbf{n}=\mathbf{3 0}\end{array}$ & $\begin{array}{c}\text { USA } \\
\mathbf{n}=100\end{array}$ & $\begin{array}{c}\text { JAPAN } \\
\mathbf{n}=\mathbf{1 0 0}\end{array}$ & $\begin{array}{c}\text { CHINA } \\
\mathbf{n}=100\end{array}$ \\
\hline $\begin{array}{l}\text { Ausländer unter den } \\
\text { Topmanagern (in \% } \\
\text { aller Topmanager) }\end{array}$ & 9,0 & 2,0 & 18,0 & - & 3,3 & 5,0 & 1,0 & - \\
\hline $\begin{array}{l}\text { Ausländer aus einem } \\
\text { fremden Sprach- und } \\
\text { Kulturraum (in \% aller } \\
\text { Topmanager) }\end{array}$ & 4,0 & 2,0 & 6,0 & - & 3,3 & 2,0 & 1,0 & - \\
\hline $\begin{array}{l}\text { Einheimische mit } \\
\text { Auslandserfahrung } \\
\text { von bis zu zwei Jahren } \\
\text { in Studium und Beruf } \\
\text { (in \% der einheimi- } \\
\text { schen Topmanager) }\end{array}$ & 16,5 & 10,2 & 5,5 & 6,0 & - & 2,1 & 2,0 & 6,0 \\
\hline $\begin{array}{l}\text { Einheimische mit } \\
\text { Auslandserfahrung } \\
\text { von mehr als zwei Jah- } \\
\text { ren in Studium und } \\
\text { Beruf (in \% der ein- } \\
\text { heimischen Topmana- } \\
\text { ger) }\end{array}$ & 19,8 & 7,9 & 13,4 & 14,0 & 10,0 & 7,4 & 32,3 & 8,0 \\
\hline
\end{tabular}

Dabei darf man die erheblichen Differenzen zwischen den einzelnen Ländern allerdings nicht übersehen. Während in China und Italien kein einziger Ausländer den Sprung an die Spitze geschafft hat, in Japan und Spanien nur jeweils einer, und das nur aufgrund einer Fusion zwischen einem einheimischen und einem ausländischen oder der Beherrschung eines einhei-

3 Die Angaben zu den Topmanagern sind für Deutschland, Frankreich, Großbritannien und die USA vom Verfasser zusammen mit Studierenden in einem Lehrforschungsprojekt ermittelt und für diesen Beitrag kontrolliert sowie gegebenenfalls korrigiert bzw. ergänzt worden, für Italien und Spanien vom Verfasser allein, für Japan von einer Diplomandin, Frau Gromer, und für China von einer Doktorandin, Frau Zhu, recherchiert worden. 
mischen durch einen ausländischen Konzern, ${ }^{4}$ in Frankreich auch gerade einmal zwei, sind es in den USA immerhin schon fünf, in Deutschland neun und in Großbritannien sogar 18. Zählt man nur die wirklichen Ausländer, also jene aus einem fremden Sprach- und Kulturraum, bleiben in den USA allerdings nicht mehr als zwei der fünf Topmanager übrig, in Deutschland vier von neun, also wie in den USA knapp die Hälfte, und in Großbritannien sogar nur gerade einmal ein Drittel, ganze sechs von $18 .{ }^{5}$ Berücksichtigt man außerdem, dass von diesen fast alle einem Unternehmen vorstehen, dass binational ist, also vor allem britisch-holländisch wie Shell, Unilever, Reckitt-Benckiser und Corus, dann fällt es noch schwerer, von einer spürbaren Internationalisierung der Wirtschaftselite zu reden. Trotz dieser Einschränkung ist Großbritannien eines der zwei Länder, neben Deutschland, für die man diesen Begriff bezogen auf den Anteil der Ausländer im Spitzenmanagement überhaupt sinnvoll verwenden kann.

Ein günstigeres Bild bietet sich, wenn man den Blick nicht auf die Anzahl der Ausländer, sondern auf die Auslandserfahrungen der einheimischen Topmanager richtet, die Transnationalität des Topmanagements. ${ }^{6}$ Da liegen die Prozentsätze in der Regel doch deutlich höher. So kann selbst im schlechtesten Fall, hier in den spanischen und den US-Unternehmen, zumindest jeder zehnte Spitzenmanager auf eine solche Erfahrung zurückblicken. In China ist es jeder siebte, in Frankreich, Großbritannien und Italien ungefähr jeder fünfte und in Deutschland und Japan sogar jeder dritte. Bis auf Frankreich, wo die kurzen Aufenthalte von bis zu insgesamt maximal zwei Jahren vorherrschen, dominieren dabei die längeren Auslandserfahrungen von mehr als zwei Jahren. Das trifft ganz besonders auf Japan zu. Dort kann fast jeder dritte Topmanager auf eine längere Phase im Ausland verweisen, zu fast 80 Prozent in Form einer beruflichen Tätigkeit in einer Tochtergesellschaft in den USA. So gut wie niemand war dagegen nur für eine kurze Zeit im Ausland. Das ist in Deutschland, der zweiten Hochburg der Auslandsaufenthalte, anders. Hier halten sich kurze und lange Aufenthalte mit 16,5 und 19,8 Prozent fast die Waage. China ähnelt in diesem Punkt, allerdings auf niedrigerem Niveau, strukturell eher Deutschland, Großbritannien und Italien eher Japan.

Es gibt offensichtlich nicht einmal erkennbare regionale Muster. Das gilt für Asien ebenso wie für Europa oder die angelsächsischen Länder. So eng China und Japan beispielsweise zusammen liegen, wenn es um die Zahl der Ausländer in den Topetagen der Wirtschaft geht, so weit liegen sie bei den langjährigen Auslandsaufenthalten auseinander. Großbritannien und die USA trennt sogar in beiderlei Hinsicht eine sehr große Kluft. Deutschland schließlich hat mit den anderen kontinentaleuropäischen Ländern ebenfalls kaum etwas gemein.

Um abschätzen zu können, ob es zumindest eine spürbare Entwicklung in Richtung Interund Transnationalität der Wirtschaftselite gibt, d.h. bei den jüngeren Spitzenmanagern ein erkennbar höheres $\mathrm{Ma} ß$ an Internationalität existiert als bei den älteren, kann man die Topmanager in zwei Alterskohorten unterteilen, eine der bis einschließlich 1949 geborenen und eine der ab 1950 geborenen. Die zweite Kohorte unterscheidet von der ersten vor allem, dass sie einen großen Teil ihrer beruflichen Karriere bereits unter den Bedingungen der seit den 1980er Jahren rasant voranschreitenden Globalisierung der Märkte absolviert hat. Es ist eine naheliegende Vermutung, dass diese veränderten Rahmenbedingungen auch die Zahl der Aus-

4 In Japan ist das Carlos Ghosn, der PDG von Renault, das 44 Prozent der Aktien von Nissan besitzt, und in Spanien Jean-Dominique Comolli, der Chef von Altadis, dem aus der Fusion der französischen Seita, dessen PDG Comolli zuvor war, und der spanischen Tabacalera entstandenen spanisch-französischen Tabakkonzern.

5 Aufgrund der Traditionen des britischen Commonwealth ist zudem fraglich, ob man bei den anderen zwölf mit Ausnahme derer, die in den USA geboren sind, überhaupt so einfach von Ausländern sprechen kann.

6 Unter Transnationalität wird hier im Unterschied zur Internationalität, die die nationale Zusammensetzung des Topmanagements ausdrückt, die internationale Erfahrung der einheimischen Spitzenmanager verstanden. 
länder in den Chefetagen der Großkonzerne und das Ausmaß wie den Umfang der Auslandserfahrungen bei den einheimischen Spitzenmanagern spürbar positiv beeinflusst haben.

Betrachtet man zunächst die Altersstruktur der ausländischen Topmanager in jenen drei Ländern, die einen Vergleich überhaupt halbwegs sinnvoll erscheinen lassen, wo es also mehr als einen oder zwei Ausländer gibt, dann bietet sich ein überraschendes Bild. Die Ausländer sind nicht jünger, sondern ganz im Gegenteil durchweg älter als die einheimischen Spitzenmanager. Das trifft gerade auf jene beiden Länder zu, die den stärksten Internationalisierungsgrad aufweisen. In Großbritannien ist die ältere Alterskohorte um mehr als 50 Prozent überrepräsentiert, in Deutschland sogar um mehr als das Doppelte. Für einen Trend Richtung Internationalität spricht das nicht.

Dieser Eindruck erhärtet sich, wenn man die einheimischen Spitzenmanager in Hinblick auf ihre Auslandserfahrungen nach Altersgruppen differenziert. Eine durchgreifende Zunahme der Auslandsaufenthalte ist nicht zu beobachten. Nimmt man zunächst die beiden Länder mit dem höchsten Anteil auslandserfahrener Topmanager in den Blick, Deutschland und Japan, so unterscheiden sich die Alterskohorten in dieser Beziehung kaum. So ist der Anteil der deutschen Vorstandsvorsitzenden, die im Ausland studiert und / oder dort berufstätig waren, von der älteren zur jüngeren Kohorte gerade einmal um gut drei Prozent gestiegen. Ähnlich sieht es in Japan und auch in den USA aus. In Großbritannien gibt es keinen nennenswerten Unterschied zwischen den Altersgruppen, in Frankreich ist sogar eine entgegengesetzte Entwicklung festzustellen. Die Auslandserfahrung ist rückläufig. Während von den älteren französischen PDG noch jeder vierte darauf verweisen kann, ist es unter den jüngeren nur noch jeder zehnte. ${ }^{7}$ Ein deutlicher Zuwachs an Auslandserfahrung lässt sich nur in jenen drei Ländern beobachten, Italien, Spanien und China, die im Vergleich zu den anderen fünf eher im zweiten Glied stehen und eine relativ späte Industrialisierung erfahren haben. Dort sind die jüngeren Topmanager, allerdings auf einem eher niedrigen Gesamtniveau, tatsächlich deutlich auslandserfahrener als ihre älteren Kollegen. In Italien liegt der Anteil gut doppelt, in China sogar fast fünfmal so hoch. Während nur ein chinesischer CEO von insgesamt 27 aus der älteren Kohorte auf eine Tätigkeit im Ausland zurück blicken kann, sind es in der jüngeren immerhin schon 13 von insgesamt 73. ${ }^{8}$ In Spanien gehören die Unternehmenschefs, die im Ausland waren, ausschließlich der jüngeren Altersgruppe an. Es sind allerdings auch nicht mehr als gerade einmal drei an der Zahl. Was man in fast allen Ländern sehen kann, ist eine Verschiebung hin zu den kürzeren Auslandsaufenthalten. Die jüngeren Spitzenmanager bevorzugen in erheblich größerem Maße als ihre älteren Kollegen den auf maximal zwei Jahre befristeten Aufenthalt im Ausland. Hier dürfte sich zum einen die Tatsache niederschlagen, dass ein solcher Aufenthalt immer stärker zu einem fast obligatorischen Bestandteil einer Karriere wird, zum anderen aber auch die Erfahrung, dass eine längere Abwesenheit aus dem Heimatland die Karriere nicht befördert, sondern sie eher bremst. Man ist einfach nicht vor Ort, wenn in den Zentralen der Mutterkonzerne über die Nachfolge für hohe Managementpositionen entschieden wird, und man verliert den Draht zu den wichtigen Netzwerken.

Das hat Konsequenzen für die Frage nach der Transnationalität des Spitzenmanagements. Will man ihren realen Umfang abschätzen, darf man die die kurzen Auslandsaktivitäten nämlich nicht wirklich mitrechnen. Da tatsächliche Transnationalität, d.h. eine halbwegs intensive Vertrautheit mit fremden Kulturen, in einer so kurzen Zeitspanne nicht einmal annähernd zu erreichen ist, kann man in dieser Beziehung nur die Anzahl der Inländer mit längeren Aus-

7 Die Differenz zu den in Hartmann (2008: 250) angegebenen Werten für die PDG resultiert aus Nachrecherchen anhand neuerer Informationsquellen.

8 Dabei ist allerdings zu berücksichtigen, dass die in der Volksrepublik China lange Jahrzehnte gültigen drastischen Reisebeschränkungen die Möglichkeit eines Auslandsstudiums oder einer Auslandstätigkeit für die ältere Generation erheblich stärker eingeschränkt haben als für die jüngere. 
landsaufenthalten berücksichtigen. ${ }^{9}$ Dann aber zeigt sich, dass die jüngere Managerkohorte in der Mehrzahl der Länder, vor allem in Deutschland und Großbritannien, den beiden Ländern mit dem insgesamt höchsten Grad an Inter- und Transnationalität, ein geringeres Maß an Transnationalität aufzuweisen hat als die ältere. Für eine in näherer Zukunft spürbar zunehmende Transnationalisierung des Topmanagements spricht das nicht gerade.

Wie stabil die nationalen Rekrutierungsmuster bei den Topmanagern sind und wie langsam die Veränderungen erfolgen, zeigt auch ein Blick zurück in das Jahr 1995 und die Entwicklung von 2005 bis heute. Wenn man die Spitzenmanager der deutschen, französischen, britischen und US-amerikanischen Großkonzerne aus den Jahren 1995 und 2005 anschaut, ${ }^{10}$ dann fällt sofort ins Auge, dass es in diesem vier Ländern sehr unterschiedliche Entwicklungen gegeben hat. Während in Frankreich das zuvor schon niedrige Niveau der Internationalisierung noch einmal gesunken ist, in Großbritannien und den USA keine tiefgreifenden Veränderungen zu sehen sind, hat sich in Deutschland in dieser Beziehung doch einiges getan. ${ }^{11}$ Zunächst zu Frankreich. Die Zahl der Ausländer unter den PDG ist mit gerade einmal zwei konstant geblieben, der Prozentsatz der Einheimischen mit Auslandserfahrung ist sogar etwas rückläufig, von knapp 23 auf nur noch gut 18 Prozent. Vor allem die längeren Auslandsaufenthalte haben spürbar von über 14 auf knapp acht Prozent abgenommen. In den USA ist ebenfalls nur eine leichte Veränderung zu erkennen, allerdings in die entgegengesetzte Richtung. So hat sich die Zahl der Ausländer unter den CEO von drei auf fünf erhöht und der Anteil der CEO mit Erfahrungen im Ausland hat sich von gut sieben auf gut neun Prozent erhöht. In den britischen Großunternehmen zeigen sich deutlich stärkere Verschiebungen. Auf der einen Seite hat sich die Anzahl der ausländischen Spitzenmanager mehr als verdoppelt, von sieben auf 18, auf der anderen der Prozentsatz der einheimischen mit Auslandserfahrung um ein Drittel reduziert, von knapp 27 auf nur noch knapp 19 Prozent. Diese auf den ersten Blick widersprüchliche Entwicklung erklärt sich zu einem nicht unbeträchtlichen Teil daraus, dass in den traditionellen binationalen Unternehmen wie Anglo American (britisch-südafrikanisch), Rio Tinto (britischaustralisch), Shell oder Unilever (beide britisch-niederländisch) üblicherweise ein ständiger Wechsel an der Spitze zwischen Briten und der jeweils anderen Nationalität erfolgt. Wenn ein Brite durch einen Nichtbriten ersetzt wird, bedeutet das angesichts der Karrierewege so gut wie immer, dass ein Brite mit Auslandserfahrung aus der Statistik verschwindet. Zusammen genommen bleibt deshalb nur eine kleine Zunahme an Inter- und Transnationalität von knapp 34 auf 37 Prozent.

Allein in Deutschland bietet sich ein gänzlich anderer Eindruck. Nicht nur hat sich die Zahl der Ausländer unter den Vorstandsvorsitzenden mehr als vervierfacht, von zwei auf neun, auch unter den deutschen Vorstandschefs sind die Auslandserfahrenen deutlich auf dem Vormarsch. Ihr Anteil ist innerhalb eines Jahrzehnts immerhin von gut 26 auf über 36 Prozent gestiegen. Allerdings ist hier ebenfalls eine Einschränkung nötig. Die Auslandsaufenthalte werden auch bei den Deutschen eher kürzer. Die insgesamt zu konstatierende Zunahme beruht fast ausschließlich darauf, dass sich die auf bis zu zwei Jahre begrenzten Auslandserfahrungen von 9,5 auf 16,5 Prozent fast verdoppelt haben. Trotz dieser Einschränkung bleibt abschließend

9 Dass selbst bei langjähriger Berufstätigkeit im Ausland nicht zwangsläufig eine Integration in die andere Kultur erfolgt, zeigt sehr anschaulich Meier am Beispiel deutscher Banker in der Londoner City (Meier 2006). Daher bleibt auch fraglich, was der hohe Prozentsatz japanischer Topmanager mit längerem Auslandsaufenthalt angesichts eines im Durchschnitt nur 3,4 Jahre dauernden und so gut wie ausschließlich in Tochtergesellschaften des eigenen Unternehmens erfolgenden Auslandseinsatzes über ihre Transnationalität tatsächlich aussagt.

10 Für diese vier Länder gibt es umfassende Vergleichswerte aus einer älteren Studie (Hartmann 1999); für Japan sind sie unvollständig, decken sich, soweit vorhanden, aber mit der Entwicklung in den genannten vier Ländern, für die anderen drei Länder fehlen sie völlig.

11 Detaillierte Zahlen in Hartmann 2008: 247. 
aber festzuhalten, dass Deutschland und Großbritannien die einzigen Länder unter den acht großen Wirtschaftsmächten sind, bei denen man überhaupt von einer spürbaren Inter- bzw. Transnationalisierung des Topmanagements sprechen kann. Bei den anderen sechs kann davon keine Rede sein.

Umso bemerkenswerter ist, dass sich in den letzten Jahren selbst in Deutschland die Internationalisierung erst einmal nicht weiter fortgesetzt hat. Das spricht auch für die nächsten Jahre eher für Kontinuität als für raschen Wandel. Seit 2005 hat es in den 100 größten Unternehmen zwar drei Neubesetzungen mit ausländischen Vorstandsvorsitzenden (dem Österreicher Peter Löscher bei Siemens, dem Dänen Caspar Rorsted bei Henkel und dem Franzosen Alain Caparros bei REWE) gegeben, im gleichen Zeitraum sind aber gleich vier ausländische Vorstandschefs ausgeschieden und von deutschen Nachfolgern ersetzt worden (der Niederländer Harry Roels bei RWE, der Österreicher Siegfried Goll bei der Zahnradfabrik Friedrichshafen, der Italiener Alessandro Bianchi bei Boehringer Ingelheim und der Norweger Björn Jansli beim Gerling Konzern). Selbst wenn man den neuen Vorstandsvorsitzenden von SAP, Leo Apotheker, als Sohn polnischer Juden in Aachen geboren, in Deutschland, Belgien und Israel aufgewachsen, deutscher Staatsbürger, aber mit erstem Wohnsitz in Paris und nach eigenen Worten ein „Weltbürger“, noch zu den drei neuen Ausländern hinzurechnet, ist keine Veränderung festzustellen. Vier Ausländer sind neu dazu gekommen, vier im gleichen Zeitraum gegangen.

\section{Die unangefochtene Vorherrschaft nationaler Karrierewege}

Warum sich der Grad an Inter- und Transnationalität bei den Spitzenmanagern der führenden Industrieländer in engen Grenzen hält und auch keine schnelle Änderung absehbar ist, wird verständlich, wenn man sich die Karrieren dieser Manager näher anschaut. Dann zeigt sich die nach wie vor ungebrochene Dominanz der traditionellen nationalen Karrieremuster und -systeme mit all ihren oft bereits seit langen Jahrzehnten gültigen Regeln. Schon ein erster oberflächlicher Blick auf die Karrierewege der Topmanager macht das sehr deutlich (siehe Tabelle 2).

Unterscheidet man die Karrieren nach den vier Grundtypen, der Hauskarriere, der Branchenkarriere, der Interbranchenkarriere und der Karriere mit Karriereabschnitten außerhalb der Wirtschaft, ${ }^{12}$ bietet sich ein ganz und gar uneinheitliches Bild. In Japan z.B. ist die Hauskarriere fast obligatorisch. Über 90 Prozent der Firmenpräsidenten haben ihr gesamtes Berufsleben in ein und demselben Unternehmen verbracht. Mit großem Abstand folgen dann Deutschland, Italien und Spanien mit Anteilen zwischen 40 und 50 Prozent. In China, Frankreich und Großbritannien sind es demgegenüber nicht einmal 20 Prozent. Dafür hat nahezu die Hälfte der chinesischen CEO und der französischen PDG einen mehr oder minder langen Teil ihrer Berufskarriere in staatlichen Institutionen, vor allem der Verwaltung oder der Politik, absolviert. In den anderen Ländern mit Ausnahme Spaniens, wo auch gut jeder vierte den Weg über die hohe Verwaltung und Politik gegangen ist, trifft das dagegen auf kaum einen Manager zu. Die Werte schwanken gerade einmal zwischen null und sechs Prozent. Reine Branchenkarrieren wiederum findet man am häufigsten in Großbritannien, wo nahezu jeder zweite Spitzenmanager seine gesamte Karriere in nur einer Branche verbracht hat. Einzig die USA weisen mit 33 Prozent noch einen halbwegs vergleichbaren Anteil auf. In Japan und Spanien liegt er demgegenüber bei weniger als zehn Prozent. Karrieren über die Branchengrenzen hinweg, nicht aber über die Grenzen der Wirtschaft sind ebenfalls besonders oft in britischen Großunternehmen zu beobachten, wo mehr als 40 Prozent diesen Weg gewählt haben. Der Abstand zu anderen Ländern ist allerdings nicht so ganz groß wie bei den Branchenkarrieren. Italien,

12 Die Definitionen der vier Typen in den Fußnoten zu Tabelle 2. 
Deutschland, die USA und Spanien folgen mit Prozentsätzen zwischen knapp 35 und knapp 29 Prozent. In Frankreich und Japan ist dieser Typus dagegen kaum zu finden. Nur jeder zwanzigste Spitzenmanager hat dort die Branche gewechselt.

Tabelle 2: Karrierewege der einheimischen Topmanager (Vorstandsvorsitzende, CEO, Chairmen, PDG, Presidents) der 100 größten Unternehmen Deutschlands, Frankreichs, Großbritanniens, Chinas, Japans und der USA sowie der 50 größten Unternehmen Italiens und der 30 größten Unternehmen Spaniens (in Prozent)

\begin{tabular}{|l|c|c|c|c|c|c|c|c|}
\hline & $\begin{array}{c}\mathbf{D} \\
\mathbf{n = 9 1}\end{array}$ & $\begin{array}{c}\mathbf{F} \\
\mathbf{n = 9 2}\end{array}$ & $\begin{array}{c}\text { GB } \\
\mathbf{n = 7 7}\end{array}$ & $\begin{array}{c}\mathbf{I T} \\
\mathbf{n = 4 6}\end{array}$ & $\begin{array}{c}\mathbf{E S P} \\
\mathbf{n = 2 9}\end{array}$ & $\begin{array}{c}\text { USA } \\
\mathbf{n = 9 1}\end{array}$ & $\begin{array}{c}\text { JAPAN } \\
\mathbf{n = 9 4}\end{array}$ & $\begin{array}{c}\text { CHINA } \\
\mathbf{n = 1 0 0}\end{array}$ \\
\hline Hauskarriere $^{13}$ & 49,5 & 17,4 & 13,0 & 45,7 & 41,4 & 36,3 & 90,4 & 16,0 \\
\hline Branchenkarriere $^{14}$ & 19,8 & 28,3 & 45,5 & 13,0 & 6,9 & 33,0 & 3,2 & 28,0 \\
\hline $\begin{array}{l}\text { Interbranchenkarrie- } \\
\text { re }^{15}\end{array}$ & 28,6 & 5,4 & 41,5 & 34,8 & 24,1 & 28,6 & 6,4 & 14,0 \\
\hline $\begin{array}{l}\text { Karriere mit Karriere- } \\
\text { abschnitten außerhalb } \\
\text { der Wirtschaft }\end{array}$ & 2,2 & 48,9 & - & 6,5 & 27,6 & 2,2 & - & 42,0 \\
\hline
\end{tabular}

Ändert man die Perspektive und betrachtet nicht die Verteilung der einzelnen Karrieretypen über die acht Länder, sondern den typischen Karriereweg in den einzelnen Ländern, ist ebenfalls eine Vielzahl von Mustern zu erkennen. Allerdings liegen Deutschland und Italien sowie Frankreich und China in diesem Falle jeweils relativ nahe beieinander. In Deutschland und Italien dominiert eindeutig die Hauskarriere mit einem Anteil von knapp der Hälfte, gefolgt von der Interbranchenkarriere mit einem guten Viertel bzw. einem Drittel und der Branchenkarriere. Völlig abgeschlagen rangiert die Karriere über die Grenzen der Wirtschaft hinaus mit nur zwei bis sechs Prozent am Ende der Skala. In Frankreich und China ist es genau umgekehrt. Dort dominiert der letztgenannte Weg, den fast jeder zweite PDG oder CEO absolviert hat, während nur jeder sechste eine Hauskarriere gemacht hat. Spanien bietet eine Mischung dieser beiden Modelle, mit einem hohen Anteil an Hauskarrieren, aber zugleich auch einem beachtlichen Teil an Karrieren mit Stationen außerhalb der Wirtschaft. In Japan gibt es neben der Hauskarriere praktisch keinen anderen Weg an die Spitze eines großen Unternehmens und Großbritannien kennt fast nur Branchen- und Interbranchenwechsler. Am ausgeglichensten ist vielleicht die Verteilung in den USA, wo sich die Topmanager fast gleichstark auf die drei innerhalb der Wirtschaft verlaufenden Karrieren verteilen.

Bei einer Untergliederung in die zwei Alterskohorten ändert sich an diesen spezifischen nationalen Karrieretypen kaum etwas. Die Veränderungen bleiben alles in allem im Rahmen der bisherigen Muster. In Deutschland verliert die Hauskarriere in der jüngeren Generation ungefähr ein Zehntel ihres Anteils und die Interbranchenkarriere gewinnt um fast die Hälfte dazu. Auch in den USA fällt der Rückgang bei den Hauskarrieren ähnlich hoch aus. Gewinner

13 Hauskarriere bedeutet, dass die jeweilige Person innerhalb der ersten fünf Jahre nach Schul- oder Hochschulabschluss in dem Unternehmen angefangen hat, das es zum Zeitpunkt der Untersuchung leitete.

14 Branchenkarriere bedeutet, dass die jeweilige Person abgesehen von der Berufsfindungsphase in den ersten fünf Jahre nach Schul- oder Hochschulabschluss immer in derselben Branche tätig war.

15 Interbranchenkarriere bedeutet, dass die jeweilige Person abgesehen von der Berufsfindungsphase in den ersten fünf Jahre nach Schul- oder Hochschulabschluss in verschiedenen Branchen, aber nicht außerhalb der Wirtschaft tätig war.

16 Karriere mit Karriereabschnitten außerhalb der Wirtschaft bedeutet, dass die jeweilige Person mehrere Jahre hauptberuflich außerhalb der Wirtschaft tätig war, vor allem in der hohen Verwaltung und in der Politik. 
ist dort aber die Branchenkarriere. In Großbritannien dagegen legt die Hauskarriere stark zu, allerdings von einem sehr niedrigen Ausgangsniveau von deutlich unter zehn Prozent. Dasselbe gilt auch für China, bei einem etwas höheren Ausgangswert. In China nimmt zudem das Gewicht der Branchenkarriere um ein gutes Viertel zu. Verlierer ist die Karriere mit Abschnitten außerhalb der Wirtschaft. Sie büßt gut ein Viertel ihres Gewichts ein, obwohl sie mit einem Anteil von über 38 Prozent auch unter den jüngeren CEO immer noch den dominierenden Karrieretyp stellt. Damit unterscheidet sich China von den beiden anderen Ländern, in denen Spitzenkarrieren nicht ausschließlich innerhalb der Wirtschaft verlaufen. In Spanien und vor allem in Frankreich gewinnt dieser Weg nämlich noch an Bedeutung. In Spanien steigt sein Anteil von einem Viertel auf ein Drittel, in Frankreich sogar von unter 45 auf über 60 Prozent. Frankreich und Spanien sind damit die einzigen Länder, in denen die traditionellen Spezifika der nationalen Karrierewege noch ausgeprägter werden. In den meisten Ländern ist es eher umgekehrt. Dort verlieren sie, wenn auch nur sehr langsam, an Gewicht.

So uneinheitlich das Bild bei den beruflichen Karrierewegen ist, so einheitlich ist es bei der davor gelagerten und für die spätere Karriere ganz zentralen Phase des Studiums. Das gilt für die Hochschulsysteme wie für die Verteilung der Topmanager auf die Hochschulen. Was den ersten Punkt angeht, so existieren nur zwei Grundtypen. Die eine Hälfte der Länder kennt traditionelle Elitehochschulen, die andere nicht. Zur ersten Gruppe zählen Frankreich mit seinen berühmten Grandes Écoles, vor allem der ENA, der École Polytechnique und der HEC, Großbritannien mit seinen Jahrhunderte alten Eliteuniversitäten Oxford und Cambridge, die USA mit ihren weltbekannten Elitehochschulen, vor allem denen der Ivy League mit Harvard, Princeton und Yale an der Spitze, und Japan mit den sogenannten „Big Five“, zu denen die beiden ältesten staatlichen Universitäten des Landes, die kaiserlichen Universitäten von Tokio (Todai) und Kyoto (Kyodai), und die beiden ältesten und berühmtesten Privatuniversitäten (Keio und Waseda) gehören. In Deutschland, Italien, Spanien und China ${ }^{17}$ gibt es diese Zweiteilung nicht.

Was nun das Studium der Spitzenmanager dieser Länder betrifft, so ist zunächst festzuhalten, dass der Anteil der ohne ein Studium in ihre Position gelangten Personen überall nur zwischen 10 und 20 Prozent liegt. ${ }^{18}$ Bei den Managern mit Hochschulexamen gibt es wie bei den nationalen Hochschulsystemen wieder eine klare Zweiteilung. In allen Ländern mit Elitehochschulen hat ungefähr die Hälfte der Spitzenmanager eine dieser Hochschulen besucht (siehe Tabelle 3). ${ }^{19}$ Am niedrigsten ist ihr Anteil in Großbritannien mit nur gut 46 Prozent, am höchsten in Frankreich mit fast zwei Dritteln. Dabei ist allerdings zu berücksichtigen, dass die Konzentration in Frankreich, Großbritannien und Japan ausgeprägter ausfällt als in den USA. Während in diesen Ländern knapp ein Drittel (Großbritannien) bis zwei Fünftel der Topma-

17 In China kann man in den letzten Jahren die Entstehung eines dem japanischen Modell verwandten Systems von Elitehochschulen (mit den beiden Pekinger Universitäten Tsinghua und Peking University an der Spitze) beobachten. Für die heutige Generation von Topmanagern spielt das aber noch keine Rolle, sondern erst für zukünftige Generationen. Ganze 14 der untersuchten Topmanager waren auf einer der sechs in den Rankings führenden Universitäten. Das sind weniger, als in Deutschland auf die ersten sechs (Aachen, München, Münster etc.) entfallen.

18 Obwohl die Werte für die USA und Großbritannien diesen Bereich nach unten wie nach oben durchbrechen, dürfte es auch dort nicht viel anders sein. Wenn man in Großbritannien die renommierte und anspruchsvolle Ausbildung zum Wirtschaftsprüfer an den Instituts of Chartered Accountants bei den Hochschulabschlüssen mit berücksichtigt, liegt man bei maximal 15 Prozent ohne Studium. In den USA dagegen dürften aufgrund der sehr hohen Anzahl und der sehr unterschiedlichen Leistungsanforderungen nicht alle Hochschulen das für die anderen Länder übliche Ausbildungsniveau erreichen (Hartmann 2005; Schreiterer 2008), so dass der sehr hohe Wert von fast 100 Prozent die Realität doch etwas verzerrt.

19 Von den Managern mit Studium sind es sogar fast zwei Drittel. 
nager (Frankreich) ${ }^{20}$ allein an den zwei renommiertesten Institutionen studiert haben, liegt dieser Prozentsatz in den USA mit weniger als 15 Prozent erheblich darunter.

Tabelle 3: Bildungsabschlüsse der einheimischen Topmanager (CEO, Chairmen, PDG, Presidents) der 100 größten Unternehmen Frankreichs, Großbritanniens, Japans und der USA (in Prozent)

\begin{tabular}{|l|c|c|c|c|}
\hline & $\begin{array}{c}\mathbf{F} \\
\mathbf{n = 9 8}\end{array}$ & $\begin{array}{c}\text { GB } \\
\mathbf{n = 8 2}\end{array}$ & $\begin{array}{c}\text { USA } \\
\mathbf{n = 9 5}\end{array}$ & $\begin{array}{c}\text { JAPAN } \\
\mathbf{n = 9 9}\end{array}$ \\
\hline Top-Eliteuniversitäten $^{21}$ & 52,1 & 31,7 & 23,2 & 52,5 \\
\hline übrige Eliteuniversitäten $^{22}$ & 12,2 & 14,6 & 27,4 & - \\
\hline Andere Universitäten $^{23}$ & 17,3 & 24,8 & 48,5 & 22,3 \\
\hline Kein Studium/ ohne Angaben $^{23}$ & 18,4 & 28,9 & 1,0 & 22,2 \\
\hline
\end{tabular}

In den anderen vier Ländern dominieren ebenso eindeutig zwei Fachgebiete, die Wirtschaftsund die Ingenieurwissenschaften (siehe Tabelle 4). Eines dieser beiden Fächer haben zwischen 60 und 80 Prozent der Topmanager studiert. Nimmt man die wenigen Naturwissenschaftler noch hinzu, steigt der Wert auf durchschnittlich drei Viertel an. Die anderen Studienfächer kommen demgegenüber nicht einmal auf 15 Prozent. Das verwundert vor allem bei den Juristen, einer früher in vielen Ländern auch in der Wirtschaft sehr einflussreichen Berufsgruppe. ${ }^{24}$ Die Rechtswissenschaften sind nur noch in Spanien von Bedeutung, wo immerhin gut jeder vierte Topmanager ein Juraexamen gemacht hat.

20 Von den PDG der 25 größten französischen Konzerne kommen mit 18 sogar fast drei Viertel von den ENA und der Polytechnique.

21 Das sind die für Frankreich die ENA, die Ecole Polytechnique und die HEC, für Großbritannien Oxford und Cambridge, für die USA die acht Hochschulen der Ivy League und für Japan die ,großen Fünf", Todai, Kyodai, Waseda, Keio und Hitotsubashi.

22 Das sind für Frankreich die anderen bekannten Grandes Écoles wie die Sciences Po (ohne anschließenden Besuch der ENA) oder die École Centrale, für Großbritannien die drei bekannten Londoner Universitäten und die vier schottischen Traditionsuniversitäten, für die USA die neben den Ivy League Hochschulen traditionell führenden 12 privaten und staatlichen Eliteuniversitäten wie Stanford oder Berkeley.

23 Bei den britischen Topmanagern sind hier auch die Absolventen der angesehenen Instituts of Chartered Accountants aufgeführt, die fast die Hälfte dieser Kategorie ausmachen.

24 Das gilt besonders für Deutschland mit seinem bis in die 1950er Jahre gültigen Juristenmonopol im höheren Dienst. Vgl. dazu Hartmann 1956 und Hartmann 1990. 
Tabelle 4: Bildungsabschlüsse aller einheimischen Topmanager (Vorstandsvorsitzende, CEO, Chairmen, Presidents) der 100 größten Unternehmen Deutschlands und Chinas sowie der 50 größten Unternehmen Italiens und der 30 größten Unternehmen Spaniens (in Prozent)

\begin{tabular}{|l|c|c|c|c|}
\hline & $\begin{array}{c}\mathbf{D} \\
\mathbf{n = 8 5 ^ { 2 5 }}\end{array}$ & $\begin{array}{c}\mathbf{I T} \\
\mathbf{n = 4 6}\end{array}$ & $\begin{array}{c}\mathbf{E S P} \\
\mathbf{n = 2 9}\end{array}$ & $\begin{array}{c}\text { CHINA } \\
\mathbf{n = 8 5}\end{array}$ \\
\hline Wirtschaftswissenschaften & 38,8 & 45,7 & 24,1 & 41,2 \\
\hline $\begin{array}{l}\text { Ingenieur- / Naturwissenschaf- } \\
\text { ten }\end{array}$ & 34,1 & 23,9 & 37,9 & 41,2 \\
\hline Jura & 10,6 & 8,7 & 27,6 & 3,5 \\
\hline Anderes Studienfach & 3,5 & 6,5 & - & 9,4 \\
\hline Ohne Studium & 12,9 & 15,2 & 10,4 & 10,6 \\
\hline
\end{tabular}

Angesichts des relativ einheitlichen Musters, das sich bei den Studiengängen und -abschlüssen der Spitzenmanager zeigt, ist es erst einmal verwunderlich, wie unterschiedlich die darauf aufbauenden beruflichen Karrieren ausfallen. Man hätte zumindest bei den Absolventen der Elitehochschulen gleichmäßigere Karrierepfade vermuten können.

Dass dem nicht so ist, demonstriert unübersehbar, wie stark der Zugang zu den Spitzenpositionen der Wirtschaft von der Struktur des gesamten Elitebildungs- und -rekrutierungssystems bestimmt wird. In Japan sieht das aber vollkommen anders aus als in Frankreich, Großbritannien oder den USA. Am erstaunlichsten ist dabei der große Unterschied zwischen Japan und Frankreich; denn die Eliten dieser beiden Länder haben viel gemein. Das gilt nicht nur für die zentrale Rolle, die die führenden Elitehochschulen bei ihrer Ausbildung spielen, sondern auch für die traditionell engen Verbindungen zwischen den Spitzen der drei entscheidenden gesellschaftlichen Sektoren, der Wirtschaft, der Politik und der Verwaltung. Was sie aber grundlegend unterscheidet, das ist die Art und Weise, in der die Toppositionen in den großen Konzernen besetzt werden. In Japan herrscht ein striktes Modell des innerbetrieblichen Aufstiegs vor. Die Absolventen der Elitehochschulen treten unmittelbar nach dem Abschluss des Studiums in ein Unternehmen ein und verlassen dieses während ihres gesamten Berufslebens nicht mehr, weil der berufliche Aufstieg ganz überwiegend vom Senioritätsprinzip bestimmt wird (Ernst 1998; Okazaki-Ward 1993). In Frankreich dagegen ist der Wechsel aus der hohen Verwaltung in die Topetage eines Unternehmens Gang und Gäbe (Hartmann 2007: 99ff). Es gibt mit dem Begriff „Pantouflage“ sogar ein eigenes Wort dafür. Immerhin fast ein Drittel der untersuchten PDG ist diesen Weg gegangen. Sie sind nach dem Studium an einer der beiden berühmtesten Grandes Écoles, der ENA und der Polytechnique, nicht direkt in ein Unternehmen eingetreten, sondern erst einmal in eines der berühmten Grands Corps, die Eliteinstitutionen der staatlichen Verwaltung. Von dort sind sie dann nach einigen Jahren in die Wirtschaft gegangen, manchmal direkt an die Spitze eines Großkonzerns, häufiger zunächst an die Spitze eines Tochterunternehmens oder in eine hohe Führungsposition in der Muttergesellschaft, etwa als Direktor für Finanzen oder Marketing. Rechnet man jene Manager hinzu, die zwar in keinem Grand Corps waren, dafür aber in einer anderen hohen Verwaltungsposition, etwa als Direktor eines Ministerialkabinetts, dann ist sogar fast jeder zweite PDG ein Wechsler aus der hohen Verwaltung. In Japan gibt es zwar auch etwas Vergleichbares, ebenfalls mit einem eigenen Wort, „Amakudari“, bezeichnet. Der Weg führt dort aber nicht bis an die Spitze der

25 Die Zahl entspricht nicht der Gesamtheit der Manager, weil hier auf die Kategorie „ohne Angabe“ im Unterschied zur Tabelle 3 verzichtet wird. In Tabelle 3 kommt es auf den Besuch von Eliteuniversitäten an. Da zählt jede Person und man kann zudem davon ausgehen, dass eine fehlende Angabe auch bedeutet, dass die Person zumindest an keiner Elitehochschule war. In Tabelle 4 geht es dagegen nur um die Verteilung auf die Studienfächer, die in ihrer Aussagekraft durch die wenigen fehlenden Personen nicht beeinflusst wird. 
großen Unternehmen, sondern nur in hohe Managementpositionen (Colignon / Usui 2003: 60ff). ${ }^{26}$

In Großbritannien sind solche Wechsel demgegenüber extrem selten. Obwohl die hohen Beamten des Civil Service den Besuch der Eliteuniversitäten wie auch den der vorgelagerten Privatschulen, der renommierten Public Schools, ${ }^{27}$ mit den Topmanagern gemein haben, jeweils die Hälfte hat eine Public School und die Hälfte der hohen Beamten und knapp ein Drittel der Spitzenmanager danach Oxford oder Cambridge besucht (Hartmann 2007: 111ff), bleiben die Sektorgrenzen so gut wie undurchdringlich. In den USA ist das reine Berufsbeamtentum aufgrund der sehr stark politisierten Verwaltung bei Weitem nicht so ausgeprägt. Der Wechsel in die hohe Verwaltung erfolgt aber weniger aus der Wirtschaft als aus der Politik. Aus Spitzenpositionen in der Wirtschaft geht man dafür aber relativ regelmäßig in die Politik. Seit dem Ende des Zweiten Weltkriegs hat durchschnittlich jedes vierte Mitglied des Kernkabinetts ${ }^{28}$ zuvor an der Spitze eines der 500 größten Unternehmen des Landes gestanden. (Hartmann 2009: 295 f). ${ }^{29}$ Den Weg zurück nehmen allerdings nur wenige. Das zeigen die Beispiele von Cheney und Paulson, die nach dem Ende der Bush-Regierung ihre alten Positionen an der Spitze von Halliburton bzw. Goldman Sachs nicht wieder einnahmen. Die großen Differenzen zwischen diesen vier Ländern zeigen, dass die Elitehochschulen zwar überall eine entscheidende Rolle spielen, aber in ein Gesamtsystem der Elitebildung und -rekrutierung eingebettet sind, das spezifischen nationalen Traditionen gehorcht und letztlich für sehr unterschiedliche nationale Karrierewege sorgt.

Dasselbe kann man auch bei den vier Ländern ohne Elitebildungseinrichtungen beobachten. Während in China aufgrund der Tatsache, dass 90 der 100 größten Unternehmen dem Staat gehören, eine in Teilen innerhalb des staatlichen Sektors (Verwaltung, Politik, Militär) verlaufende Karriere immer noch sehr häufig anzutreffen ist, spielt dieser Weg in Deutschland und Italien überhaupt keine Rolle. Hier dominiert die Hauskarriere im Unternehmen. Spanien kennt beide Muster. Die Hauskarriere hat fast dasselbe Gewicht wie in Deutschland und Italien. Gleichzeitig spielen aber auch die traditionellen, nach französischem Vorbild organisierten Elitecorps der staatlichen Verwaltung und damit eine Tätigkeit im Staatsapparat eine wichtige Rolle. Immerhin jeder fünfte Unternehmensleiter kommt von dort, wie im Übrigen auch jedes vierte Kabinettsmitglied (Alvarez de Cienfuegos 1999; Hartmann 2007: 102ff).

Dass solch unterschiedliche nationale Elitebildungs- und -rekrutierungssysteme mit ihren jeweils spezifischen Karrierepfaden und Elitenetzwerken einer Inter- und Transnationalisierung der Wirtschaftseliten große Hindernisse in den Weg legen, ist offensichtlich. Am Beispiel Frankreichs lässt sich schön zeigen, wie eine Stärkung dieser nationalen Systeme den Prozess der Inter- und Transnationalisierung zeitweise sogar wieder umkehren kann. Vergleicht man die beiden Alterskohorten unter den PDG, dann fällt ein Zusammenhang sofort auf. In der jüngeren Kohorte liegt der Anteil der Absolventen von ENA, Polytechnique und HEC um über zehn Prozent höher als in der älteren. Gleichzeitig steigt der Prozentsatz der zu Beginn der Karriere in einem Grand Corps tätigen PDG bei den jüngeren verglichen mit ihren älteren

26 Demgegenüber ist ein Wechsel aus der hohen Verwaltung in die Regierungskabinette in beiden Ländern üblich.

27 Die berühmteste von ihnen ist ohne Zweifel Eton.

28 Zum Kernkabinett werden hier neben dem Präsidenten und dem Vizepräsidenten die Minister für Inneres, Finanzen und Wirtschaft sowie für Äußeres und Verteidigung gerechnet, insgesamt 121 Personen seit 1945.

29 In Großbritannien kommt ein solcher Wechsel dagegen nur ganz ausnahmsweise vor. Einen der sehr seltenen Fälle stellt Mervyn Davies dar, Staatssekretär im Kabinett Brown, der zuvor CEO und Chairman bei der Großbank Standard Chartered war. Der aktuelle CEO dieser Bank, Peter Sands, bildet ebenfalls eine Ausnahme, wenn auch in anderer Richtung. Er hat seine Karriere im Außenministerium begonnen, um dann über Mc Kinsey zu Standard Chartered zu gelangen.. 
Kollegen um fast ein Viertel. Der Karrieretypus der Pantouflage nimmt in der jüngeren Altersgruppe dementsprechend ebenfalls um ein Viertel zu. All das erklärt, warum Frankreich das einzige Land ist, in dem in der jüngeren Managerkohorte ein spürbar geringeres Maß an transnationalen Karriereverläufen zu konstatieren ist. Während von den älteren PDG jeder vierte auf Auslandserfahrung zurück blicken kann, gilt das nur noch für jeden zehnten jüngeren PDG. ${ }^{30}$

Nicht nur in Frankreich, sondern auch in Großbritannien und den USA gilt außerdem, dass Ausländer die beste Aussicht auf die Führung eines Großkonzerns haben, wenn diese Position auf dem Wege einer unternehmensinternen Karriere erreicht wird. Hauskarrieren sind unter den ausländischen Topmanagern in diesen drei Ländern deutlich überrepräsentiert. Das trifft in besonderem Maße auf die USA zu, wo vier der fünf Ausländer in diese Kategorie fallen. Aber auch in Großbritannien sind Hauskarrieren unter den Ausländern mit einem Anteil von über 25 Prozent wesentlich stärker vertreten als unter den einheimischen Topmanagern. Letztere sind dementsprechend bei den Branchen- und Interbranchenkarrieren deutlich überrepräsentiert. Diese Tatsache erklärt sich in erster Linie aus den persönlichen Verbindungen und Netzwerken, die aus dem Besuch einer Elitebildungseinrichtung resultieren. Sie erleichtern den Wechsel über Unternehmens- und Branchengrenzen hinweg, weil nicht das Unternehmen für das erforderliche gegenseitige Verständnis und Vertrauen sorgen, sondern die gemeinsame Schul- oder Hochschulzeit bzw. der mit den Eliteinstitutionen allgemein verknüpfte Ruf und Habitus. Daher ist es nicht verwunderlich, dass die US-CEO, die in Harvard studiert haben, die Einzigen sind, die im Rahmen ihrer beruflichen Karriere über die Grenzen der Wirtschaft hinaus tätig waren und insgesamt auch zu über zwei Dritteln eine über die Firmen- und Branchengrenzen hinausgehende Karriere gemacht haben. Nur ein gutes Viertel hat dagegen eine reine Hauskarriere absolviert. Bei den übrigen $\mathrm{CEO}$ zeigt sich eine andere Verteilung. Fast 40 Prozent haben eine Hauskarriere und nur gut 60 Prozent eine über Unternehmens- und Branchengrenzen hinausreichende Karriere gemacht. Wer nicht an einer solchen Eliteeinrichtung war, hat es einfach deutlich schwerer, ist stärker auf den Aufstieg innerhalb eines Unternehmens mit seinen stärker intern geprägten Regeln und Netzwerken angewiesen. Das demonstriert auch ein Vergleich der Alterskohorten. So liegt der Anteil der Ivy-League-Absolventen in der jüngeren Kohorte der US-CEO um über ein Drittel höher als in der älteren. Gleichzeitig sinkt der Prozentsatz der Hauskarrieren um ein Zehntel. Diese Regel, von der nur Japan mit seinem sehr strikten System der lebenslangen Unternehmenszugehörigkeit abweicht, bestätigt sich auch, wenn man die Länder ohne Elitehochschulen betrachtet. In Deutschland, dem einzigen Land unter ihnen mit mehr als einem Ausländer an der Spitze eines Großunternehmens, gibt es keinen Unterschied in den Karrieremustern zwischen In- und Ausländern.

\section{Fazit}

Das Spitzenmanagement der führenden multinationalen Konzerne ist weder inter- noch transnational. Das ist in aller Kürze das Resümee der Analyse. Nur jeder zwanzigste Topmanager kommt aus dem Ausland und nur knapp jeder vierte einheimische Spitzenmanager kann auf Auslandserfahrungen während seines Studiums oder im Laufe seiner Berufskarriere zurückblicken. Länger im Ausland, d.h. mehr als zwei Jahre, war sogar nur jeder siebte. Die anderen

30 Gerade bei Frankreich mit seinem „Königsweg“ über die Grandes Écoles und die Grands Corps gilt es allerdings zu berücksichtigen, dass der Kohortenvergleich stets zwei Effekte enthält, den eines im Zeitverlauf sich verändernden Karrieremusters und den des schnelleren Aufstiegs durch einen bestimmten Karrieretypus, in diesem Falle die Pantouflage. Für die Frage nach der Inter- und Transnationalität des Topmanagements spielt diese Differenz jedoch keine nennenswerte Rolle. Ob die Pantouflage generell an Bedeutung gewonnen hat oder nur zu einer schnelleren Karriere verhilft, in beiden Fällen heißt das: das traditionelle nationale Karrieremuster erweist sich als deutlich durchsetzungsfähiger. 
waren nur so kurz dort, dass man von einem Kennenlernen fremder Kulturen nicht wirklich sprechen kann. Die Unterschiede zwischen den einzelnen Ländern sind dabei allerdings enorm. Während in China und Italien überhaupt kein Ausländer an der Spitze eines Großkonzerns steht, es in Frankreich, Japan, Spanien und den USA auch nur zwischen einem und fünf gibt, sind es in Deutschland und Großbritannien immerhin neun bzw. 18. Ausländer aus einem fremden Sprach- und Kulturraum gibt es allerdings generell nur selten. Die Prozentsätze schwanken gerade einmal zwischen null und sechs Prozent. Bei den Auslandserfahrungen der einheimischen Topmanager gibt es ebenfalls große Differenzen. Hier liegen die deutschen Vorstandsvorsitzenden mit einem Anteil von mehr als einem Drittel vorn, gefolgt von den Japanern. Am Ende rangieren mit weniger als zehn bzw. gerade einmal 14 Prozent die USAmerikaner und die Chinesen. Vergleicht man die ältere, vor 1950 geborene mit der jüngeren, ab 1950 geborenen Generation der Spitzenmanager, zeigt sich keine nennenswerte Tendenz hin zu mehr Inter- oder Transnationalität bei den jüngeren Topmanagern. In Frankreich ist sogar ein gegenläufiger Trend zu beobachten. Alles in allem kein Anlass, von einer globalen oder transnationalen Wirtschaftselite oder Klasse zu sprechen.

Der entscheidende Grund für das geringe Ausmaß an Inter- und Transnationalität ist die ungebrochene Dominanz traditioneller nationaler Karrieremuster. Überall erreicht man die Spitze eines der führenden Großunternehmen am ehesten, wenn man diesen traditionellen Pfaden folgt. So sollte man in den Ländern, die Elitehochschulen haben (Frankreich, Großbritannien, Japan und die USA), eine von diesen auch besucht haben. In Frankreich und China ist es zudem günstig, die berufliche Karriere zunächst im staatlichen Sektor zu beginnen, um erst später in die Wirtschaft zu wechseln. Fast jeder zweite Topmanager hat es so gemacht. In den meisten anderen Ländern ist das dagegen ein Weg, der nur ganz ausnahmsweise an die Spitze eines Großkonzerns führt. Dort dominieren entweder die Hauskarrieren in einem einzigen Unternehmen oder branchen- bzw. branchenübergreifende Karrieren innerhalb der Wirtschaft. Es gibt also selbst in den führenden multinationalen Konzernen kein allgemein gültiges und Erfolg versprechendes transnationales Karrieremuster.

Nun könnte es sein, dass die Konzentration der Analyse auf die absoluten Spitzenpositionen den Blick auf die unterhalb dieser Ebene sich abspielenden Prozesse verschleiert oder vernebelt. Dort könnte die Entwicklung durchaus schon deutlich weiter voran geschritten sein. Diese Vermutung liegt erst einmal nahe, erweist sich bei näherem Hinsehen aber nichtsdestotrotz als falsch. Wenn man die Nationalität und die Karrierewege der ca. 400 einfachen Vorstandsmitglieder der 100 größten deutschen Unternehmen betrachtet, zeigt sich bei ihnen vielmehr ganz im Gegenteil sogar ein deutlich geringeres Maß an Transnationalität als bei den Vorstandsvorsitzenden. Der Anteil der Ausländer liegt mit zehn Prozent zwar geringfügig höher, der Anteil der Inländer mit Auslandserfahrung dafür mit nur gut 20 gegenüber mehr als 36 Prozent aber erheblich niedriger. ${ }^{31}$ Dazu kommt, dass die jüngere Generation der Vorstandsmitglieder ebenfalls den kurzen Auslandsaufenthalt bevorzugt. Bei einem, wie unter den jüngeren Vorstandsvorsitzenden, insgesamt leicht höheren Prozentsatz an Auslandserfahrung überwiegt bei den jüngeren Vorstandsmitgliedern der kurze Aufenthalt im Ausland in einem Verhältnis von drei zu zwei. Bei ihren älteren Kollegen ist es genau umgekehrt. Bei ihnen dominieren die mehr als zwei Jahre dauernden Auslandsaufenthalte im Verhältnis von zwei zu eins. Insgesamt spricht auch das nicht für einen spürbar zunehmenden Grad an Transnationalität.

Geht man noch eine Ebene tiefer in der Hierarchie und wirft einen Blick auf die Gesamtheit der Führungskräfte, ändert sich das Bild ebenfalls nicht. Wie Pohlmann und Bär in einer aktuellen Studie am Beispiel von sechs Großunternehmen der Automobil-, Metall-, Chemie- und

31 Das ändert sich auch dann nicht, wenn man jene ca. 100 Vorstandsmitglieder unberücksichtigt lässt, deren Karriereverläufe nicht zur Gänze geklärt werden konnten. Dann verringert sich die Differenz zwar um ein Drittel, bleibt aber immer noch ausgesprochen groß. 
Software-Branche zeigen, bewegt sich der Anteil der Ausländer an allen Führungskräften dieser Firmen gerade einmal zwischen knapp einem und gut sechs Prozent. Rechnet man die Österreicher und Schweizer heraus, sinkt er sogar auf Werte zwischen knapp einem halben und gut zwei Prozent. Einzig ein Sportartikelhersteller, der für seine internationalen Produktionsketten bekannt ist, kommt auf deutlich höhere Anteile (Pohlmann/Bär 2009: Schaubild 2.2).

So eindeutig all diese empirischen Forschungsergebnisse auch sind, sie bedeuten nicht, dass es überhaupt keine Tendenz hin zu mehr Transnationalität gibt, und auch nicht, dass soziale Ungleichheit nicht zunehmend von einer transnationalen Perspektive aus zu betrachten ist. Sie mahnen nur zur Vorsicht bei der Begriffsbildung, bei der Interpretation empirischer Befunde und bei der Erstellung von allgemeinen Prognosen für die Zukunft. Nationalstaaten und alle damit verbundenen Strukturen und Elemente nationaler Ungleichheits- und Herrschaftssysteme weisen ein sehr viel stärkeres Beharrungsvermögen auf, als manche vorschnelle Verallgemeinerung einzelner Beobachtungen vermuten lässt.

Wer aus qualitativen Studien mit einer Fallzahl von oft nur wenigen Personen ${ }^{32}$ generelle Trendaussagen ableitet, mag auf lange Sicht zwar richtig liegen, auf lange Sicht dürfte aber einen Zeitraum von etlichen Jahrzehnten und mehr bedeuten. Schon jetzt von transnationalen Eliten oder Klassen zu sprechen, erlauben solche Studien auf keinen Fall, zumal wenn man berücksichtigt, dass das Aufkommen neuer Wirtschaftsmächte wie China und Indien die Bedeutung nationaler Karrieremuster und nationaler Eliten auf globaler Ebene erst einmal verstärken dürfte. Eine ähnliche Kritik trifft, in allerdings deutlich geringerem Maße, auch für Untersuchungen über die ,,interlocking directors“ wie die von Carroll zu. In den Boards nehmen die Anzahl der Ausländer und die Anzahl der transnationalen Verbindungen zweifellos zu, zumindest in Europa und Nordamerika. Das gilt aber in erster Linie für jene Personen, die dort Aufsichtsfunktionen nach dem Muster der deutschen Aufsichtsräte wahrnehmen. Die im Tagesgeschäft aktiven Topmanager, die die Konzerne tatsächlich führen, werden von dieser Entwicklung bislang, wie gesehen, nur wenig berührt. Sie aber bilden den wirklichen Maßstab für die Inter- und Transnationalisierung der Wirtschaftselite, denn sie müssen tatsächlich in dem Land arbeiten und leben, in dem die Konzernzentrale angesiedelt ist. Als Ausländer müssen sie sich daher mit für sie fremden Kulturen auch wirklich vertraut machen. Für die Aufsichtsräte gilt das nicht. Sie müssen für die in der Regel nur sehr begrenzte Anzahl von Sitzungen pro Jahr keine derartige Transnationalität an den Tag legen. Sie sorgen zwar für eine Stärkung grenzüberschreitender Kontakte, für die Ausbildung von stabilen und die nationalen Karrieremuster spürbar verändernden transnationalen Netzwerken reicht das aber bei weitem nicht aus.

Griffige und leicht eingängige Formulierungen wie die von Castells ,elites are cosmopolitan, people are local“ (Castells 1996: 415) klingen zwar gut, die Realität erfassen sie jedoch bestenfalls ansatzweise. Praktisch bedeutet das auch, dass die Begründung der hohen Managergehälter mit den Gesetzen des transnationalen Marktes für Spitzenmanager nach wie vor mit der Realität nicht viel zu tun hat. ${ }^{33}$ Sie dient ganz eindeutig vor allem dem Zweck, diese auf veränderten gesellschaftlichen Machtverhältnissen beruhenden, exorbitanten Einkommen öffentlich zu rechtfertigen.

32 Solche Studien sind typisch für die derzeitige Diskussion über Transnationalität (vgl. u.a. die Beiträge in Kreutzer / Roth 2006 oder Nowicka 2006.).

33 Bei einzelnen hoch spezialisierten und hochqualifizierten Berufsgruppen wie etwa den Investmentbankern kann das anders aussehen. Hier kann die Aussage von den globalen Arbeitsmärkten mehr Realitätsgehalt haben. 


\section{Literatur}

Alvarez de Cienfuegos, Ignacio Molina (1999): Spain. Still the Primacy of Corporatism?, in: Edward C. Page / Vincent Wright (Hrsg.), Bureaucratic Elites in Western European States, Oxford, S. 32-54

Beck, Ulrich (2008): Jenseits von Klasse und Nation: Individualisierung und Transnationalisierung sozialer Ungleichheiten, in: Soziale Welt 59, S. 301-325.

Carroll, William K. (2009): Transnationalists and national networkers in the global corporate elite, in: Global Networks 9, S. 289-314.

Castells, Manuel (1996): The Rise of the Network Society. The Information Age: Economy, Society, and Culture, Cambridge.

Colignon, Richard A. / Chikako Usui (2003): Amakudari. The Hidden Fabric of Japan's Economy, Ithaca.

Dahrendorf, Ralf (2000): Die globale Klasse und die neue Ungleichheit, in: Merkur 54, S. 1057-1068.

Dahrendorf, Ralf (2009): Die Derivatisierung der Welt und ihre Folgen. Ein Gespräch mit Ralf Dahrendorf zum 80. Geburtstag, in: Leviathan 37, S. 177-186.

Ernst, Angelika (1998): Aufstieg - Anreiz - Auslese. Karrieremuster und Karriereverläufe von Akademikern in Japan, Opladen.

Hartmann, Heinz (1956): Der zahlenmäßige Beitrag der deutschen Hochschulen zur Gruppe der industriellen Führungskräfte, in: Zeitschrift für die gesamte Staatswissenschaft 112, S. 144-163.

Hartmann, Michael (1990). Juristen in der Wirtschaft - eine Elite im Wandel, München.

Hartmann, Michael (1999): Auf dem Weg zu einer transnationalen Bourgeoisie?, in: Leviathan 27, S. 113-141.

Hartmann, Michael (2005): Studiengebühren und Hochschulzugang: Vorbild USA? in: Leviathan 33, S. 439-463.

Hartmann, Michael (2007): Eliten und Macht in Europa, Frankfurt / Main.

Hartmann, Michael (2008): Transnationale Klassenbildung? In: Peter A. Berger / Anja Weiß (Hrsg.), Transnationalisierung sozialer Ungleichheit, Wiesbaden, S. 241-258

Hartmann, Michael (2009): Politische Elite und Einkommensverteilung in den USA seit 1945, in: Leviathan 37, S. 281-304.

Kanther, Rosabeth Moss (1995): World Class. Thriving Locally in the Global Economy, New York.

Kreutzer, Florian / Silke Roth (Hrsg.) (2006): Transnationale Karrieren. Biografien, Lebensführung und Mobilität, Wiesbaden.

Meier, Lars (2006): Den Ort spüren, Distanz erfahren - Irritationen der alltäglichen Handlungen deutscher Finanzbeschäftigter in London, in: Florian Kreutzer / Silke Roth (Hrsg.), Transnationale Karrieren. Biografien, Lebensführung und Mobilität, Wiesbaden, S. 224-239.

Nowicka, Magdalena (2006): Transnational Professionals and their Cosmopolitan Universes, Frankfurt / Main.

Okazaki-Ward, Lola (1993): Management Education and Training in Japan, London.

Pohlmann, Markus / Stefan Bär (2009): Zur Frage von Internationalität und Exklusivität - Elitereproduktion und soziale Schließung?, Vortrag an der Universität Heidelberg am 28.6.2009.

Schreiterer, Ulrich (2008): Traumfabrik Harvard. Warum amerikanische Hochschulen so anders sind, Frankfurt / Main. 
Sklair, Leslie (2001): The Transnational Capitalist Class, Oxford.

Yoo, Taeyoung / Soo Hee Lee (2009): In Search of Social Capital in State-Activist Capitalism: Elite Networks in France and Korea, in: Organization Studies 30, S. 529-547.

Prof. Dr. Michael Hartmann Technische Universität Darmstadt Institut für Soziologie Residenzschloß 64283 Darmstadt Hartmann@ifs.tu-darmstadt.de 